\title{
WB89 520: A Small Isolated High Mass Star Forming Region Associated with an Extremely Metal-Poor Nebula
}

\author{
Jun-Jie Wang, Jing-Yao Hu, Jian-Yan Wei \\ National Astronomical Observatory, Beijing, 100012, P.R. China \\ Leonardo Testi \\ Osservatorio Astrofisico di Arcetri, Largo E. Fermi 5, I-50125 Firenze, \\ Italy
}

\begin{abstract}
In this paper, by discussing and analyzing the observational results of near infrared imaging, optical spectroscopy and ${ }^{12} \mathrm{CO}$ molecular line, together with IRAS and NVSS data, we conclude that WB89 520 is an UC HII region with a very young compact stellar cluster. The spectrum of the UC HII region shows that it is an extremely metal-poor nebula, which has the lowest line ratio of $[\mathrm{NII}] / \mathrm{H}_{\alpha}(\sim 1 / 56)$ discovered in the nebulae of our Galaxy until now. We give two possible explanations for the low metallicity.
\end{abstract}

\section{Introduction}

It is well-known that high mass OB stars form in Giant Molecular Clouds (GMCs) which lie in the spiral arms. And generally the mean diameter of a GMC is 45 pc with a mass of $10^{5-6} M_{\odot}$ (Blitz,1987).

WB89 520 ( IRAS 04000+5052) has not been studied extensively before. In 1993, based on the analysis of near infrared photometry and optical CCD imaging, Wang et al. (1993) found the source of IRAS $04000+5052$ is an interesting near infrared variable object associated with an HII region ( 1993 ).

\section{Observations and Discussions}

The near infrared imaging observations were carried out on Nov.7 1995, using the $1.5 \mathrm{~m}$ TIRGO telescope with the Arcetri near-infrared camera (ARNICA) at Gornergrat Observatory. The ARNICA detector is a NICMOS3 $256 \times 256$ pixel HgCdTe array. The scale on the detector is roughly $0.95 \% /$ pixel. WB89 520 was observed in the $\mathrm{J}, \mathrm{H}$ and $\mathrm{K}$ broad-bands and $\mathrm{H}_{2} \mathrm{~S}(1) 1-0, \mathrm{Br}_{\gamma}$ narrow bands. Spectroscopic observations of this source were taken with the Beijing Astronomical Observatory $2.16 \mathrm{~m}$ telescope and the OMR spectrograph with a Tektronix $1024 \times 1024 \mathrm{CCD}$ as detector. The $200 \AA \mathrm{Amm}^{-1}$ grating was used, blazed at $6500 \AA$.

A very strong $\mathrm{H}_{\alpha}$ emission line appears in the optical spectrum. And the continuum of the spectrum shows that WB89 520 is an extremely red source. 
The spectral type of the embedded exciting star of the UCHII region can be obtained using the Lyman-continuum photon method. The radio continuum flux at $1.4 \mathrm{GHz}$ is used to calculate the number of Lyman-continuum photons heating the gas. i.e.

$$
N(\mathrm{rad})=7.54 \times 10^{46}(\nu / G H z)^{0.1} \times\left(T_{e} / 10^{4} K\right)^{-0.45}\left(S_{\nu} / J y\right)(D / k p c)^{2}
$$

Here we used an electron temperature $10^{4} \mathrm{~K}$ like many others used ([6],[8] etc.), and assume the kinematic distance $3.88 \mathrm{kpc}$ obtained from $\mathrm{CO}$ observation [9]; thus $\log \mathrm{N}(\mathrm{rad})$ can be calculated to be 46.27 , corresponding to the spectral type B0.5 in Panagia's table ( 1973 ).

WB89 520 appears to be a single somewhat extended red source on optical CCD images. But from our infrared imaging observations, an infrared cluster, including 15 infrared detectable objects, is discovered. It is a very small $(0.83$ $\mathrm{pc}(\mathrm{NS}) \times 1.55 \mathrm{pc}(\mathrm{EW}))$ and isolated high mass star forming region in which there is a B0.5 type star exciting the UC HII region. Possibly it is not an onarm high mass star forming region and is located far beyond the Perseus arm. Its heliodistance $(\sim 4.29 \mathrm{kpc})$ is twice as far as that of the Perseus arm, which means the OB stars are forming not only in GMCs of the spiral arms.

The spectrum of the UC HII region shows that it is an extremely metal-poor nebula, which has the lowest line ratio of $[\mathrm{NII}] / \mathrm{H}_{\alpha}(\sim 1 / 56)$ discovered in the nebulae of our Galaxy until now. We suppose that there are two possibilities to explain this point. One is that the material constituting this nebula does not belong to our Galaxy, and comes from some extragalactic objects due to some physical mechanisms, for instance merging or accretion of galaxies. Another explanation is that in the outer disk without spiral arm in our Galaxy some primeval material or some primeval material without over-contamination by objects of inner Galaxy still exists. For both the two explanations we need more examples like WB89 520 and need to have further observational evidences and theoretical results.

\section{References}

Blitz, L.,1987, Physical processes in interstellar clouds. Morfill and Scholer, eds., (Reidel:Dordrecht), p.35

Panagia, N., 1973, AJ, 78, 929

Wang, J.-J., Qian, Z.-Y., Hu, J.-Y., Jiang, B.-W., Wang, G., 1993, Chin. Astron. Astrophys., 17, no. 1, 61 